\title{
A combination of methylprednisolone and quercetin is effective for the treatment of cardiac contusion following blunt chest trauma in rats
}

\author{
F. Demir ${ }^{1}$, A. Güzel ${ }^{2}$, C. Katı ${ }^{3}$, C. Karadeniz ${ }^{4}$, U. Akdemir ${ }^{3}$, A. Okuyucu ${ }^{5}$, A. Gacar 6 , \\ S. Özdemir ${ }^{2}$ and T. Güvenç ${ }^{6}$ \\ ${ }^{1}$ Department of Pediatric Cardiology, Faculty of Medicine, Dicle University, Diyarbakır, Turkey \\ ${ }^{2}$ Department of Pediatrics, Faculty of Medicine, Ondokuz Mayıs University, Samsun, Turkey \\ ${ }^{3}$ Department of Emergency Medicine, Faculty of Medicine, Ondokuz Mayıs University, Samsun, Turkey \\ ${ }^{4}$ Pediatric Cardiology Services, Behçet Uz Children's Hospital, Izmir, Turkey \\ ${ }^{5}$ Department of Medical Biochemistry, Faculty of Medicine, Ondokuz Mayıs University, Samsun, Turkey \\ ${ }^{6}$ Department of Pathology, Faculty of Veterinary Medicine, Ondokuz Mayıs University, Samsun, Turkey
}

\begin{abstract}
Cardiac contusion is a potentially fatal complication of blunt chest trauma. The effects of a combination of quercetin and methylprednisolone against trauma-induced cardiac contusion were studied. Thirty-five female Sprague-Dawley rats were divided into five groups $(n=7)$ as follows: sham, cardiac contusion with no therapy, treated with methylprednisolone (30 mg/kg on the first day, and $3 \mathrm{mg} / \mathrm{kg}$ on the following days), treated with quercetin $\left(50 \mathrm{mg} \cdot \mathrm{kg}^{-1} \cdot \mathrm{day}^{-1}\right.$ ), and treated with a combination of methylprednisolone and quercetin. Serum troponin I (Tn-I) and tumor necrosis factor-alpha (TNF- $\alpha$ ) levels and cardiac histopathological findings were evaluated. Tn-I and TNF- $\alpha$ levels were elevated after contusion $(P=0.001$ and $P=0.001)$. Seven days later, Tn-I and TNF- $\alpha$ levels decreased in the rats treated with methylprednisolone, quercetin, and the combination of methylprednisolone and quercetin compared to the rats without therapy, but a statistical significance was found only with the combination therapy $(P=0.001$ and $P=0.011$, respectively). Histopathological degeneration and necrosis scores were statistically lower in the methylprednisolone and quercetin combination group compared to the group treated only with methylprednisolone $(P=0.017$ and $P=0.007$, respectively). However, only degeneration scores were lower in the combination therapy group compared to the group treated only with quercetin $(P=0.017)$. Inducible nitric oxide synthase positivity scores were decreased in all treatment groups compared to the untreated groups $(P=0.097, P=0.026$, and $P=0.004$, respectively). We conclude that a combination of quercetin and methylprednisolone can be used for the specific treatment of cardiac contusion.
\end{abstract}

Key words: Blunt chest trauma; Cardiac contusion; Quercetin; Methylprednisolone; iNOS

\section{Introduction}

Cardiac contusion is a potentially fatal complication of blunt chest trauma (1). Motor vehicle accidents are the most common cause of cardiac contusions (2), and the frequency of cardiac contusion ranges from $8 \%$ to $76 \%$ depending on the diagnostic criteria used after trauma (3). The Centers for Disease Control and Prevention (USA) reports approximately 30,000 patients with blunt cardiac trauma per year (4).

Blunt chest trauma may lead to cardiac injury including myocardial contusion, hematoma, ventricular rupture, ventricular septal defect, and valvular damage, or a decrease in coronary blood flow associated with vascular lesions, subintimal hemorrhage, intraluminal thrombosis, or vasoconstriction $(1,5)$. Because cardiac contusion may potentially cause severe hemodynamic compromise and life-threatening arrhythmias $(3,6)$, its accurate management is very important. However, current therapy for cardiac contusion is mainly supportive and includes fluid replacement and inotropic and anti-arrhythmic drugs (7); no specific treatment for myocardial injury has yet been established.

Steroids have anti-inflammatory activity and they have been shown to be beneficial in the treatment of myocardial injury associated with ischemia, cardiac surgery, and cyclosporin A (8-10). Quercetin is an antioxidant flavone, which has been found to inhibit

Correspondence: F. Demir, Department of Pediatric Cardiology, Faculty of Medicine, Dicle University, 21280, Sur, Diyarbakır, Turkey. E-mail: fikridemir@yahoo.com 
ischemia-reperfusion-induced gene and protein expression of nicotinamide adenine dinucleotide phosphate oxidase 2 (NOX2) and inducible nitric oxide synthase (iNOS) and to have beneficial effects on myocardial injury $(11,12)$. Although the combination of glucocorticoids and antioxidants has been studied in the management of various organ injuries (13-15), there are a limited number of investigations evaluating their effectiveness in cardiac lesions (16). Moreover, to date, no study has used this combination in the treatment of cardiac contusion.

This study aimed to evaluate the consequences of separate and combined use of methylprednisolone and quercetin in a rat model of myocardial injury associated with cardiac contusion.

\section{Material and Methods}

The experimental protocol was approved by the Experimental Animal Studies Ethics Committee of Ondokuz Mayis University, Samsun, Turkey.

\section{Animals and experimental protocol}

All rats were provided by the Experimental Research Center of the Medical Faculty of Ondokuz Mayis University. Thirty-five female Sprague-Dawley rats weighing 250-300 g were used as experimental subjects. They were kept under standard experimental laboratory conditions $\left(24^{\circ} \mathrm{C}, 12: 12-\mathrm{h}\right.$ dark-light cycle, free access to food and water, and $60 \%$ relative humidity).

The rats were allocated to five groups $(n=7)$ as follows: S, sham; Cont, cardiac contusion; Cont+MP, Cont group treated with ip methylprednisolone (Prednol-L; Mustafa Nevzat, Turkey) (17); Cont +QC, Cont group treated with oral gavage administration of quercetin (Sigma Chemical Co., USA) (18); Cont + MP + QC, Cont group treated with methylprednisolone and quercetin combined. All drug dosages used are shown in Table 1.

The rats were anesthetized with $100 \mathrm{mg} / \mathrm{kg}$ ketamine hydrochloride (ip) and $10 \mathrm{mg} / \mathrm{kg}$ xylazine (ip) and then placed in a supine position on plexiglass boards. A blunt chest trauma model was induced applying $1.96 \mathrm{~J}$ of impact energy (19). The impact energy (E) of the falling weight was calculated from the equation: $E=m g h(20)$, where $\mathrm{m}$ is the mass of the falling aluminum weight $(0.4 \mathrm{~kg}), \mathrm{g}$ is gravitational acceleration $\left(9.8 \mathrm{~m} / \mathrm{s}^{2}\right)$, and $\mathrm{h}$ is the height of the weight above the study platform $(50 \mathrm{~cm})$. The rats were observed following the procedure, and all of them survived.

At the end of the seventh day, all of the rats were killed following an ip ketamine hydrochloride injection. After the thoracic procedure, the hearts were removed from the thorax for histopathological and immunohistochemical investigation. Histopathological assessments were blinded and performed by an experienced pathologist.

\section{Morphological and histopathological studies of myocardial contusion}

The effects of contusion on heart tissue were evaluated by histopathological and immunohistochemical studies. The hearts were rapidly excised and fixed in $10 \%$ buffered formalin for 24-72 $\mathrm{h}$ and embedded in paraffin following a routine procedure. Sections $5-\mu \mathrm{m}$ thick were prepared from the blocks and stained with hematoxylin and eosin for routine microscopic examination. Subsequently, a blinded pathologist examined all microscopic slides. Necrosis, degeneration, hemorrhage, and calcification in myocardial tissue were scored from 0 to 3 as follows: 0 , negative; $1+$, weak; $2+$, moderate; and $3+$, severe.

\section{Immunohistochemistry procedure for myocardial contusion}

Heart tissue samples were fixed in $10 \%$ neutral buffered formalin and embedded in paraffin. All samples were sectioned at a thickness of $5 \mu \mathrm{m}$, then deparaffinized and rehydrated. The streptavidin-biotin-peroxidase complex technique (Histostain Plus Kit; Zymed, cat. No. 85-8943, USA) was used for the immunohistochemical study.

Endogenous peroxidase activity was removed by incubation with $2 \%$ hydrogen peroxidase in methanol for $30 \mathrm{~min}$ at room temperature. Antigen retrieval was performed by microwave treatment for $15 \mathrm{~min}$ in citrate buffer, pH 6.0. Rabbit polyclonal anti-iNOS antibody (1/250; Abcam, cat. No.: ab3523, UK) was used as primary antibody. Aminoethyl carbazole was used as the

Table 1. Treatment protocols in the study groups.

\begin{tabular}{|c|c|c|}
\hline Study groups & $1^{\text {st }}$ day (single dose, 5 min after contusion) & $2^{\text {nd }}-7^{\text {th }}$ days (once a day) \\
\hline S & No treatment & No treatment \\
\hline Cont & No treatment & No treatment \\
\hline Cont + MP & MP (30 mg/kg bolus, ip) & MP (3 mg/kg, ip) \\
\hline Cont + QC & $\mathrm{QC}(50 \mathrm{mg} / \mathrm{kg}, p o)$ & QC $(50 \mathrm{mg} / \mathrm{kg}, p o)$ \\
\hline Cont + MP + QC & $\begin{array}{l}\text { MP (30 mg/kg bolus, ip) } \\
\text { QC }(50 \mathrm{mg} / \mathrm{kg}, p o)\end{array}$ & $\begin{array}{c}\text { MP (3 mg/kg, ip) } \\
\text { QC (50 mg/kg, po) }\end{array}$ \\
\hline
\end{tabular}

S: Sham; Cont: cardiac contusion; MP: methylprednisolone; QC: quercetin. 
chromogen in hydrogen peroxide for $10 \mathrm{~min}$, which was controlled by visual observation with a microscope. The sections were counterstained with Lillie Mayer's hematoxylin for $1 \mathrm{~min}$ and rinsed with tap water. Subsequently, the sections were mounted with an aqueous mounting medium.

All morphological images were acquired using a light microscope (Nikon Eclipse E600, Nikon Corporation, Japan). Immunohistochemical iNOS staining of the heart tissue samples was evaluated semiquantitatively according to the intensity of the staining, which was scored as faint $(-/+)$, mild $(1+)$, moderate $(2+)$, and strong $(3+)$. Evaluation of immunostaining was performed in at least eight randomly selected areas per heart section, using two sections from each animal at $400 \times$ magnification. The final score calculated in each category for each rat was the mean of the scores from the heart sections examined.

\section{Biochemical procedure}

Blood samples taken from rat hearts 7 days after contusion were put into potassium EDTA tubes. They were centrifuged at $1500 \mathrm{~g}$ for $10 \mathrm{~min}$ in order to obtain the serum. Plasma samples were kept frozen at $-40^{\circ} \mathrm{C}$ initially and brought to $+4^{\circ} \mathrm{C}$ one night before the study. After the samples were thawed, biochemical analyses were performed. Plasma tumor necrosis factor-alpha (TNF- $\alpha$; Cat. No. KRC3011, Invitrogen, USA) and serum troponin I (Tn-I; Cat. No. CK-E30258, Hangzhou Eastbiopharm Corporation, China) levels were measured using sandwich ELISA. All biochemical results were represented as picograms per milliliter.

\section{Statistical analysis}

All measurements were analyzed with the SPSS 21.0 package software (USA). Measurements obtained were reported as median (minimum/maximum) or means $\pm S D$. All values were compared with a Mann-Whitney $U$-test. The statistical significance level was $P<0.05$.

\section{Results}

\section{Biochemical findings}

Following blunt chest trauma, serum Tn-I levels were significantly increased in the Cont group compared to the $S$ group $(P=0.001)$, and $T n-I$ levels in the treatment groups (Cont $+M P$, Cont $+Q C$, and Cont $+M P+Q C$ ) were lower than in the Cont group $(P=0.073, P=0.710$, and $P=0.001$, respectively; Table 2 ). Likewise, serum TNF- $\alpha$ levels were also significantly increased after chest trauma in the Cont group compared to the control (S group; $\mathrm{P}=0.001$ ), and $\mathrm{TNF}-\alpha$ levels in the treatment groups (Cont $+M P$, Cont $+Q C$, and Cont $+M P+Q C$ ) were lower than in the untreated group (Cont; $P=0.097$, $P=0.259$, and $P=0.011$, respectively; Table 2). Serum levels of both Tn-I and TNF- $\alpha$ were significantly lower in the Cont $+\mathrm{MP}+\mathrm{QC}$ group than in the Cont $+\mathrm{QC}$ group $(P=0.011$ and $P=0.038$, respectively); serum $\mathrm{Tn}-\mathrm{I}$ and TNF $-\alpha$ levels in the Cont $+\mathrm{MP}+\mathrm{QC}$ group were also lower than in the Cont + MP group, but the difference was statistically significant only for $\mathrm{Tn}-\mathrm{I}(\mathrm{P}=0.011$; Table 2$)$.

\section{Histopathological findings}

All findings from histopathological scores in study groups are represented in Figure 1A-D. Scores for hemorrhage, degeneration, necrosis, and calcification in myocardial tissue were greater in the Cont group than in the $S$ group $(P=0.001, P=0.001, P=0.208$, and $P=0.026$, respectively; Table 3 ). Although the only significant decrease observed in animals treated with methylprednisolone compared with the Cont group was in hemorrhage scores $(P=0.026)$ and none of the histopathological parameters changed significantly in the Cont+QC group compared to the Cont group, simultaneous treatment of contused animals with methylprednisolone and quercetin resulted in a significant decrease in degeneration, necrosis, and calcification scores compared to the Cont group $(P=0.001, P=0.007$, and $P=0.026$, respectively; Table 3 ).

Degeneration and necrosis scores were statistically lower in the Cont+MP+QC group compared to the Cont + MP group $(P=0.017$ and $P=0.007$, respectively). However, only degeneration scores were lower in the Cont + MP + QC group compared to the Cont + QC group $(P=0.017$; Table 3).

\section{Immunohistochemical results}

The iNOS myocardial expression score was significantly higher in the Cont group (median $3+$ ) than in the $S$ group (median $+1-; \mathrm{P}=0.001$; Figure $2 \mathrm{~A}$ and $\mathrm{B}$ ). iNOS positivity scores were decreased in the treatment groups (Cont $+\mathrm{MP}$, Cont $+\mathrm{QC}$, and Cont $+\mathrm{MP}+\mathrm{QC}$; median $2+$, median $2+$, and median $1+$, respectively) compared to the Cont group (median $3+; \mathrm{P}=0.097, \mathrm{P}=0.026$, and $\mathrm{P}=0.004$, respectively; Table 4, Figure 2).

Between treatment groups, iNOS expression scores were lower in the Cont $+\mathrm{MP}+\mathrm{QC}$ group (Figure 2E) compared to the other treatment groups [Cont+MP (Figure 2D) and Cont+QC (Figure 2C)], but these differences were not statistically significant $(P=0.165$ and $\mathrm{P}=0.208$, respectively; Table 4 ).

\section{Discussion}

Cardiac contusion is an important condition, which may cause myocardial injury and associated morbidity or mortality. A variety of anti-inflammatory and antioxidant agents have been studied in the management of various cardiac injury conditions $(8,9,21-23)$. The current study investigated the single and combined effects of methylprednisolone and quercetin in myocardial injury caused by cardiac contusion and found that combined use of these agents resulted in a more pronounced improvement in the 
Table 2. Comparison of serum troponin I and TNF- $\alpha$ levels in experimental groups.

\begin{tabular}{lccccc}
\hline & S & Cont & Cont + MP & Cont + QC & Cont + MP+QC \\
\hline Tn-I $(\mathrm{pg} / \mathrm{mL})$ & $223.02 \pm 30.98$ & $412.65 \pm 33.63^{\S}$ & $342.61 \pm 63.10$ & $368.51 \pm 91.06$ & $214.67 \pm 78.91^{+* \#}$ \\
TNF- $\alpha(\mathrm{pg} / \mathrm{mL})$ & $14.55 \pm 1.09$ & $17.64 \pm 1.68^{\S}$ & $16.42 \pm 5.48$ & $16.55 \pm 0.96$ & $14.56 \pm 2.05^{+\#}$ \\
\hline
\end{tabular}

Data are reported as means \pm SD. S: Sham; Cont: cardiac contusion; MP: methylprednisolone; QC: quercetin; Tn-I: troponin I; TNF- $\alpha$ : tumor necrosis factor-alpha. ${ }^{\S} \mathrm{P}<0.05$ compared to $\mathrm{S}$ group; ${ }^{+} \mathrm{P}<0.05$ compared to Cont group; ${ }^{*} \mathrm{P}<0.05$ compared to Cont $+\mathrm{MP}$ group; ${ }^{\#} \mathrm{P}<0.05$ compared to Cont $+\mathrm{QC}$ group (Mann-Whitney $\mathrm{U}$ test).

markers of inflammation and myocardial damage than monotherapy.

The diagnosis of cardiac contusion after blunt chest trauma is difficult due to the lack of standard diagnostic criteria and its varied manifestations (3). Cardiac contusion is diagnosed by a combination of medical history regarding the mechanism of trauma, together with clinical findings and diagnostic tests $(3,6)$. Blunt cardiac trauma may lead to contused myocardium (showing muscle necrosis, edema, and hemorrhage), complex arrhythmia, cardiac rupture, coronary artery laceration, pericardial laceration, shock, and sudden death $(4,24)$. The pathophysiological and molecular changes of cardiac contusion after blunt chest trauma remain unclear, and there is no specific drug for use in this condition; therefore, the current treatment strategy following cardiac contusion involves supportive and symptomatic therapy. It is thought that histopathological and molecular designed studies may play a role in the development of therapeutic strategies for cardiac contusion.

iNOS is an enzyme expressed in various tissues, including the heart, only during pathological and inflammatory conditions. Although nitric oxide generation is stated to be an adaptive mechanism against ischemia-reperfusion injury, in the presence of oxidative stress it augments the stressful condition via oxidative or nitrosative species, inhibits mitochondrial respiration, and results in deleterious effects on the myocardium (25-27). TNF- $\alpha$ is a mediator released in any inflammatory process. It has a depressor effect on the heart and causes stimulation of iNOS expression $(26,28)$. Therefore, these markers and histological findings were thought to be appropriate tools in the evaluation of recovery from myocardial injury.

In the present study, all the markers of inflammation and myocardial damage that were investigated, including TNF$\alpha$, Tn-I, and iNOS expression, and myocardial hemorrhage, degeneration, necrosis, and calcification scores were significantly lower in rats treated with combined methylprednisolone and quercetin compared to the Cont group. However, the animals treated solely with methylprednisolone had statistically lower levels of Tn-I and myocardial hemorrhage scores, whereas the only significant change provided by isolated quercetin therapy was a decrease in iNOS expression. It was found that a combination treatment with methylprednisolone and quercetin provided a more explicit decrease in indicators of inflammation and myocardial injury than separate use of these medications. It is thus
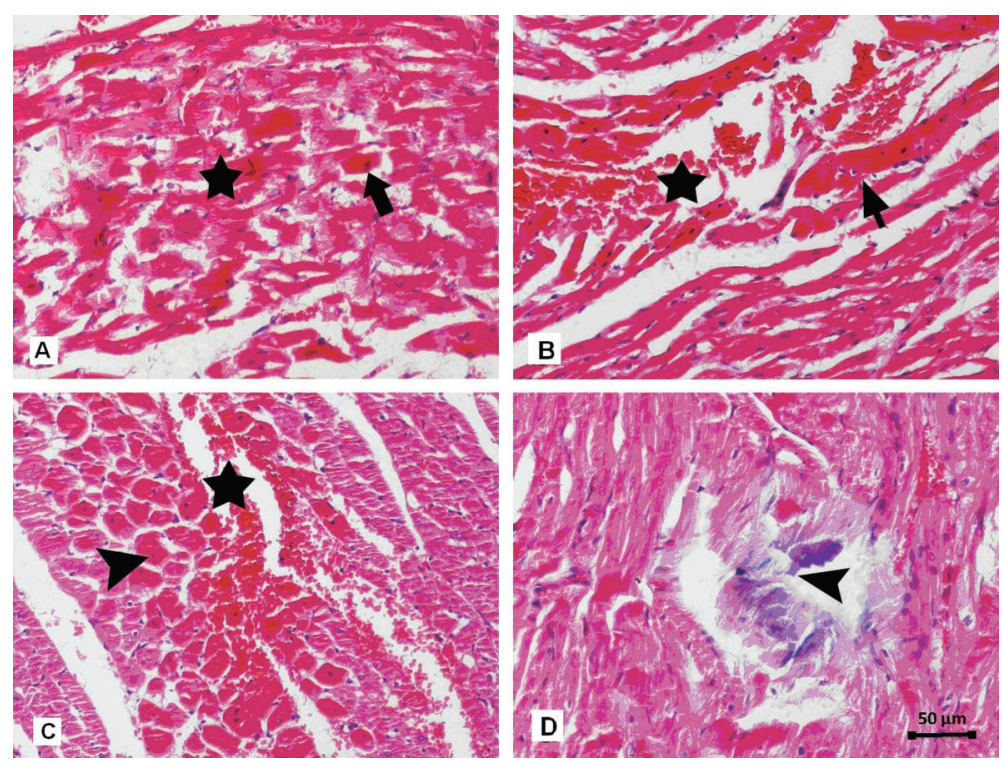

Figure 1. Histopathological evaluation of heart tissues. A, Degeneration and severe necrosis (thick arrow) of cardiac muscle with abundant karyopyknosis in Cont group. B, Longitudinal view of cardiac fibers, separated heart muscle due to excessive bleeding (star) and necrotic myofibers (arrow) in Cont group. C, Transverse view of cardiac muscle with dense eosinophilic cytoplasm and pyknotic nuclei (arrowhead) and excessive bleeding between the muscle fibers in Cont+MP group. D, Calcification area (arrowhead) surrounded with degenerative and necrotic myofibers in Cont $+Q C$ group. Cont: cardiac contusion; Cont +MP: Cont group treated with methylprednisolone; Cont $+\mathrm{QC}$ : Cont group treated with quercetin. Hematoxylin and eosin staining, $20 \times$ magnification. 
Table 3. Comparison of groups according to histopathological findings.

\begin{tabular}{lccrrl}
\hline & $S$ & Cont & Cont + MP & Cont + QC & Cont + MP+QC \\
\hline HEM & $0(0+/ 1+)$ & $1+(0+/ 1+)$ & $0(0+/ 1+)^{+}$ & $0(0+/ 2+)$ & $0(0+/ 1+)$ \\
DEG & $0(0+/ 1+)$ & $2+(2+/ 2+)^{\S}$ & $1+(1+/ 3+)$ & $1+(1+/ 2+)$ & $0(0+/ 1+)^{+* \#}$ \\
NEC & $0(0+/ 0+)$ & $2+(1+/ 3+)^{\S}$ & $2+(1+/ 3+)$ & $1+(0+/ 2+)$ & $0(0+/ 1+)^{+*}$ \\
CAL & $0(0+/ 0+)$ & $1+(0+/ 2+)^{\S}$ & $0(0+/ 1+)$ & $0(0+/ 2+)$ & $0(0+/ 0+)^{+}$ \\
\hline
\end{tabular}

Data are reported as median (minimum/maximum). S: Sham; Cont: cardiac contusion; MP: methylprednisolone; QC: quercetin; HEM: hemorrhage; DEG: degeneration; NEC: necrosis; CAL: calcification. ${ }^{\S} \mathrm{P}<0.05$ compared to $\mathrm{S}$ group; ${ }^{+} \mathrm{P}<0.05$ compared to Cont group; ${ }^{*} \mathrm{P}<0.05$ compared to Cont + MP group; ${ }^{\#} \mathrm{P}<0.05$ compared to Cont $+\mathrm{QC}$ group (Mann-Whitney $\mathrm{U}$ test).
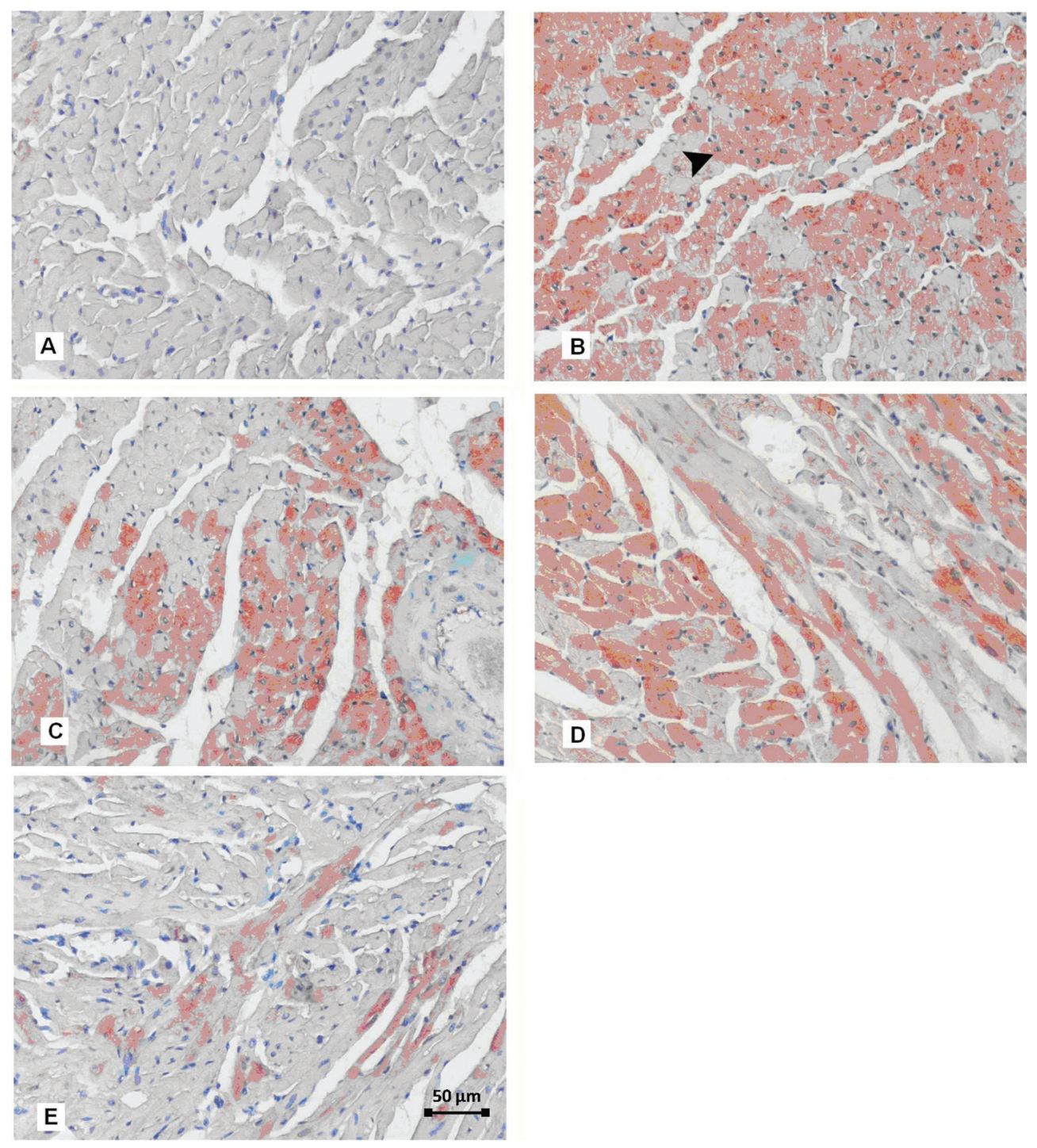

Figure 2. Immunohistochemical expression of inducible nitric oxide synthase (iNOS) in the control and treatment groups of heart muscle. $A$, There is no immunopositive reaction of iNOS in muscle fibers of $\mathrm{S}$ group. $B$, Strongly positive iNOS immunoreactivity of heart muscle (arrowhead) in Cont group. C, Moderate immunopositive reaction in Cont+QC group. D, Intermediate iNOS immunoreactivity of heart muscle in Cont + MP group. $E$, Weak immunopositive reaction of iNOS in Cont + MP + QC group. S: Sham; Cont: cardiac contusion; Cont +MP: Cont group treated with methylprednisolone; Cont +QC: Cont group treated with quercetin; Cont + MP + QC: Cont group treated with methylprednisolone and quercetin. Immunoperoxidase technique, Harris hematoxylin counter staining, 3-amino-9-ethylcarbazole (AEC) as a chromogen, $20 \times$ magnification. 
Table 4. Semiquantitative assessment of iNOS positive cells in myocardial tissues of each group.

\begin{tabular}{cccccc}
\hline & S & Cont & Cont + MP & Cont +QC & Cont + MP + QC \\
\hline iNOS & $1+(0+/ 2+)$ & $3+(2+/ 3+)^{\S}$ & $2+(1+/ 3+)$ & $2+(1+/ 2+)^{+}$ & $1+(1+/ 2+)^{+}$ \\
\hline
\end{tabular}

Data are reported as median (minimum/maximum). S: Sham; Cont: cardiac contusion; MP: methylprednisolone; QC: quercetin; iNOS: inducible nitric oxide synthase. ${ }^{\S} \mathrm{P}<0.05$ compared to $\mathrm{S}$ group; ${ }^{+} \mathrm{P}<0.05$ compared to Cont group (Mann-Whitney $\mathrm{U}$ test).

thought that methylprednisolone and quercetin synergistically increase their effectiveness.

Steroids act as anti-inflammatory agents through the reduction of early inflammatory processes such as increased capillary permeability, edema formation, and leukocyte migration, as well as suppression of stimulusdependent expression of many proinflammatory proteins through the inhibition of transcriptional pathways in target cells (29). Steroids have been found to have cardioprotective effects via various mediators including heat shock proteins, TNF- $\alpha$, interleukins, and endothelial nitric oxide synthase during inflammation $(30,31)$. Glucocorticoids have also been shown to be effective in recovery from myocardial injury associated with ischemia, cardiac surgery, and cyclosporin $A(8,9,10)$.

Quercetin exerts its antioxidant properties by scavenging reactive oxygen and nitrogen species and inhibiting xanthine oxidase activity and lipid peroxidation (32). It increases endothelial nitric oxide and induces vasodilatation through smooth muscle relaxation (33). Quercetin has been experimentally used in the treatment of ischemia and myocarditis $(23,34)$. Experimental studies have reported that quercetin protects from ischemia-reperfusion injury through the prevention of apoptosis and inhibition of the mitochondria-dependent caspase pathway in animals $(35,36)$. A combination of glucocorticoids and antioxidants has been used in various experimental organ injuries including lung, nerves, and cardiac radiofrequency lesions $(13,14,16)$. The present study differed from earlier ones

\section{References}

1. Kamdar G, Santucci K, Emerson BL. Management of pediatric cardiac trauma in the ED. Clin Pediatr Emerg Med 2011; 12: 323-332, doi: 10.1016/j.cpem.2011.09.001.

2. Patil RR, Mane D, Jariwala P. Acute myocardial infarction following blunt chest trauma with intracranial bleed: a rare case report. Indian Heart J 2013; 65: 311-314, doi: 10.1016/ j.ihj.2013.04.018.

3. El-Chami MF, Nicholson W, Helmy T. Blunt cardiac trauma. J Emerg Med 2008; 35: 127-133, doi: 10.1016/j.jemermed. 2007.03.018.

4. Elie MC. Blunt cardiac injury. Mt Sinai J Med 2006; 73: 542552.

5. Kusmierczyk M, Drohomirecka A, Michalowska I, Michalek P, Juraszynski Z, Rozanski J. Delayed diagnosis of pericardial hematoma compressing the right ventricle after blunt chest trauma. J Card Surg 2013; 28: 701, doi: through evaluation of the efficiency of these drugs in myocardial injury associated with cardiac contusion and the use of quercetin as an accompanying agent to steroids.

Cardiac contusion has a diverse clinical presentation, ranging from asymptomatic and spontaneously recovering patients to a fatal course associated with cardiogenic shock. Cases with hypotension, cardiac failure, and arrhythmia require treatment consisting of supportive measures such as fluid replacement, inotropes, and anti-arrhythmic medications $(7,37)$. However, no specific therapy for injury related to cardiac contusion has yet been established. The current study showed that glucocorticoids and antioxidants, such as quercetin, might improve myocardial recovery and that combination therapy was more effective. Further animal and human studies providing more evidence for the use of those medications as a specific treatment option in patients with cardiac contusion may be important because of the potential fatality of the condition.

In conclusion, methylprednisolone and quercetin seem to improve, to some extent, the recovery of myocardial injury associated with cardiac contusion. However, combined use of those agents provided a more dramatic improvement, possibly because of their synergistic effects. Therefore, it is possible that the combination of methylprednisolone and quercetin can be used as a specific management strategy in cardiac contusion. Nevertheless, randomized controlled human trials are warranted in order to assess these statements.
$10.1111 /$ jocs. 12137

6. Keough $\mathrm{V}$, Letizia $\mathrm{M}$. Blunt cardiac injury in the elderly trauma patient. Int J Trauma Nurs 1998; 4: 38-43, doi: 10.1016/S1075-4210(98)90045-1.

7. Malangoni MA, McHenry CR, Jacobs DG. Outcome of serious blunt cardiac injury. Surgery 1994; 116: 628-632.

8. Checchia PA, Backer CL, Bronicki RA, Baden HP, Crawford SE, Green TP, et al. Dexamethasone reduces postoperative troponin levels in children undergoing cardiopulmonary bypass. Crit Care Med 2003; 31: 1742-1745, doi: 10.1097/ 01.CCM.0000063443.32874.60.

9. Xu B, Strom J, Chen QM. Dexamethasone induces transcriptional activation of $\mathrm{Bcl}-\mathrm{xL}$ gene and inhibits cardiac injury by myocardial ischemia. Eur J Pharmacol 2011; 668: 194-200, doi: 10.1016/j.ejphar.2011.06.019.

10. Florio S, Ciarcia R, Crispino L, Pagnini U, Ruocco A, Kumar 
$C$, et al. Hydrocortisone has a protective effect on CyclosporinA-induced cardiotoxicity. J Cell Physiol 2003; 195: 21-26, doi: 10.1002/jcp.10216.

11. Boots AW, Haenen GR, Bast A. Health effects of quercetin: from antioxidant to nutraceutical. Eur J Pharmacol 2008; 585: 325-337, doi: 10.1016/j.ejphar.2008.03.008.

12. Wan LL, Xia J, Ye D, Liu J, Chen J, Wang G. Effects of quercetin on gene and protein expression of NOX and NOS after myocardial ischemia and reperfusion in rabbit. Cardiovasc Ther 2009; 27: 28-33, doi: 10.1111/j.17555922.2009.00071.x.

13. Crisafulli C, Mazzon E, Muia C, Bella P, Esposito E, Meli R, et al. Effects of combination of melatonin and dexamethasone on acute lung injury in a mice model of carrageenaninduced pleurisy. J Pineal Res 2006; 41: 228-237, doi: 10.1111/j.1600-079X.2006.00358.x.

14. Cayli SR, Kocak A, Yilmaz U, Tekiner A, Erbil M, Ozturk C, et al. Effect of combined treatment with melatonin and methylprednisolone on neurological recovery after experimental spinal cord injury. Eur Spine J 2004; 13: 724-732, doi: 10.1007/s00586-003-0550-y.

15. Ocalan K, Solak O, Esme H, Sirmali M, Dilek H, Oz G, et al. Efficacy of budesonide and interleukin-10 in an experimental rat model with isolated bilateral pulmonary contusion created by blunt thoracic trauma. Eur J Cardiothorac Surg 2013; 43: 163-167, doi: 10.1093/ejcts/ezs313.

16. Fenelon G, Franco M, Mora O, Katchburian E, de Paola AA. Combined therapy with steroids and antioxidants prevents ultrastructural damage surrounding chronic radiofrequency lesions. Pacing Clin Electrophysiol 2004; 27: 65-72, doi: 10.1111/j.1540-8159.2004.00387.x

17. Teng D, Pang QF, Yan WJ, Zhao XW, Xu CY. The harmful effect of prolonged high-dose methylprednisolone in acute lung injury. Int Immunopharmacol 2013; 15: 223-226, doi: 10.1016/j.intimp.2012.12.004.

18. El-Sayed NS, Rizk SM. The protective effect of quercetin, green tea or malt extracts against experimentally-induced lung fibrosis in rats. Afr J Pharm Pharmacol 2009; 3: 191-201.

19. Raghavendran K, Davidson BA, Helinski JD, Marschke CJ, Manderscheid P, Woytash JA, et al. A rat model for isolated bilateral lung contusion from blunt chest trauma. Anesth Analg 2005; 101: 1482-1489, doi: 10.1213/01.ANE. $0000180201.25746 .1 \mathrm{~F}$.

20. Miller PR, Croce MA, Bee TK, Qaisi WG, Smith CP, Collins $\mathrm{GL}$, et al. ARDS after pulmonary contusion: accurate measurement of contusion volume identifies high-risk patients. J Trauma 2001; 51: 223-228, doi: 10.1097/ 00005373-200108000-00003.

21. Baxter BT, Moore EE, Synhorst DP, Reiter MJ, Harken AH. Graded experimental myocardial contusion: impact on cardiac rhythm, coronary artery flow, ventricular function, and myocardial oxygen consumption. J Trauma 1988; 28: 1411-1417, doi: 10.1097/00005373-198810000-00001.

22. Liedtke AJ, Allen RP, Nellis SH. Effects of blunt cardiac trauma on coronary vasomotion, perfusion, myocardial mechanics, and metabolism. J Trauma 1980; 20: 777-785, doi: 10.1097/00005373-198009000-00012.

23. Ikizler M, Erkasap N, Dernek S, Kural T, Kaygisiz Z. Dietary polyphenol quercetin protects rat hearts during reperfusion: enhanced antioxidant capacity with chronic treatment.
Anadolu Kardiyol Derg 2007; 7: 404-410.

24. Mascaro M, Trojian TH. Blunt cardiac contusions. Clin Sports Med 2013; 32: 267-271, doi: 10.1016/j.csm. 2012.12.004

25. Jones SP, Girod WG, Palazzo AJ, Granger DN, Grisham $M B$, Jourd'Heuil D, et al. Myocardial ischemia-reperfusion injury is exacerbated in absence of endothelial cell nitric oxide synthase. Am J Physiol 1999; 276: H1567-H1573.

26. Panas D, Khadour FH, Szabo C, Schulz R. Proinflammatory cytokines depress cardiac efficiency by a nitric oxide-dependent mechanism. Am J Physiol 1998; 275: H1016-H1023.

27. Otani $\mathrm{H}$. The role of nitric oxide in myocardial repair and remodeling. Antioxid Redox Signal 2009; 11: 1913-1928, doi: 10.1089/ars.2009.2453.

28. Natanson C, Eichenholz PW, Danner RL, Eichacker PQ, Hoffman WD, Kuo GC, et al. Endotoxin and tumor necrosis factor challenges in dogs simulate the cardiovascular profile of human septic shock. J Exp Med 1989; 169: 823-832, doi: 10.1084/jem.169.3.823.

29. Rubens FD, Nathan H, Labow R, Williams KS, Wozny D, Karsh $\mathrm{J}$, et al. Effects of methylprednisolone and a biocompatible copolymer circuit on blood activation during cardiopulmonary bypass. Ann Thorac Surg 2005; 79: 655665, doi: 10.1016/j.athoracsur.2004.07.044.

30. Keski-Nisula J, Pesonen E, Olkkola KT, Peltola K, Neuvonen PJ, Tuominen N, et al. Methylprednisolone in neonatal cardiac surgery: reduced inflammation without improved clinical outcome. Ann Thorac Surg 2013; 95: 2126-2132, doi: 10.1016/j.athoracsur.2013.02.013.

31. Morimoto RI, Kline MP, Bimston DN, Cotto JJ. The heatshock response: regulation and function of heat-shock proteins and molecular chaperones. Essays Biochem 1997; 32: 17-29.

32. Frankel EN, Kanner J, German JB, Parks E, Kinsella JE. Inhibition of oxidation of human low-density lipoprotein by phenolic substances in red wine. Lancet 1993; 341: 454457, doi: 10.1016/0140-6736(93)90206-V.

33. Fitzpatrick DF, Hirschfield SL, Coffey RG. Endotheliumdependent vasorelaxing activity of wine and other grape products. Am J Physiol 1993; 265: H774-H778.

34. Milenkovic M, Arsenovic-Ranin N, Stojic-Vukanic Z, Bufan B, Vucicevic D, Jancic I. Quercetin ameliorates experimental autoimmune myocarditis in rats. J Pharm Pharm Sci 2010; 13: 311-319.

35. Shen J, Lee W, Gu Y, Tong Y, Fung PC, Tong L. Ginkgo biloba extract (EGb761) inhibits mitochondria-dependent caspase pathway and prevents apoptosis in hypoxiareoxygenated cardiomyocytes. Chin Med 2011; 6: 8, doi: 10.1186/1749-8546-6-8.

36. Arumugam S, Thandavarayan RA, Arozal W, Sari FR, Giridharan VV, Soetikno V, et al. Quercetin offers cardioprotection against progression of experimental autoimmune myocarditis by suppression of oxidative and endoplasmic reticulum stress via endothelin-1/MAPK signalling. Free Radic Res 2012; 46: 154-163, doi: 10.3109/10715762. 2011.647010.

37. Holanda MS, Dominguez MJ, Lopez-Espadas F, Lopez M, Díaz-Reganon J, Rodriguez-Borregan JC. Cardiac contusion following blunt chest trauma. Eur J Emerg Med 2006; 13: 373-376, doi: 10.1097/MEJ.0b013e32801112f6. 